\title{
Thank you to referees, 2016
}

The editors would like to thank the following people who reviewed articles in 2016:

Patrick Ainley, Davina Allen, Mi Young An, Stefan Angel, Anneli Anttonen, Lindsey Appleyard, Suengho Baek, Clare Bambra, Michael Barr, Armando Barrientos, Ben Baumberg, Daniel Béland, Lutz Bellmann, Sylvia Bergh, Derek Birrell, Anne Leonara Blaakilde, Jo Blanden, Florian Blank, Alice Bloch, Agnes Blome, Hugh Bochel, Tom Boland, Vikki Boliver, Catherine Bolzendahl, Giuliano Bonoli, Silke Bothfeld, Harriet Bradley, Jonathan Bradshaw, Michelle Brady, Thomas Bredgaard, Nicola Brimblecombe, Patrick Briwb, Tania Burchardt, Roger Burrows, Daylian Cain, Ines Calzada, Martin Caraher, Ewan Carr, Sarah Carr, Maria Casado-Diaz, Dorte Caswell, Harriet Churchill, John Clarke, Jochen Clasen, Daniel Clegg, Stuart Connor, Barbara da Roit, Hulya Dagdeviren, Catrina Denvir, David Devins, Hans Dietrich, Michelle Dion, Jeremy Dixon, Rachael Dobson, Kevin Doogan, Fiona Dukelow, Grant Duncan, Bernhard Ebbinghaus, Hans Ekbrand, Anne Lise Ellingsæter, Nick Ellison, Elisabeth Lund Engebretsen, Adalbert Evers, Kevin Farnsworth, Ross Fergusson, JoseLuis Fernandez, Emanuele Ferragina, Naomi Finch, Tony Fitzpatrick, Timo Fleckenstein, Del Roy Fletcher, John Flint, Tone Flotten, Ray Forrest, Liam Foster, Lucy Frith, Bernard Gallagher, Ludovica Gambaro, Qin Gao, Kayleigh Garthwaite, Heidi Gjersøe, Jean Golding, Ian Gough, Jose A Tapia Granados, Ann Marie Gray, Paolo Graziano, Ian Greener, Lee Gregory, Amanda Grenier, Bina Gubhaju, Karon Gush, Bjorn Gustafsson, Mia Hakovirta, Anthony Hall, Kelly Hall, Niamh Hardiman, Jamie Harding, Juho Harkonen, Jenny Harlock, Madonna Harrington Meyer, Elizabeth Harrison, Tina Haux, Richard Hazenberg, Martin Heidenreich, Kene Henkens, Paul Henman, Marianne Hester, Rod Hick, John Hills, Susan Himmelweit, Xian Huang, John Hudson, Shereen Hussein, Björn Hvinden, Mark Hyde, Rana Jawad, Kelley Johnson, Gerwyn Jones, Karen Jones, Rhys Jones, Johannes Kananen, Yuri Kazepov, Justin Keen, Jeremy Kendall, Majella Kilkey, Elina Kilpi-Jakonen, Bo-Yung Kim, Minjeong Kim, Soo Wan Kim, Yeon-Myung Kim, Trudie Knijn, Alison Koslowski, Michael Kottelenberg, Huckju Kwon, Sarah Kyambi, David Lain, Mary Larkin, Daniel Larsson, Blanche Le Bihan, Sung-Hee Lee, Bettina Leibetseder, Lutz Leisering, Thomas Leoni, Janine Leschke, Stephen Levine, Colin Lindsay, Jane Lindsay, Mark Livingston, Peter Lloyd-Sherlock, Liz Lloyd, Toby Lowe, Asa Lundqvist, Julia Lynch, Julie Macleavy, 
Lindsey Macmillan, Ilaria Madama, Mikkel Mailand, Gill Main, Margitta Mätzke, Lucy Mayblin, Aidan McGarry, Stephen McKay, Nasar Meer, Jane Millar, Nicola Moran, Rebecca Morris, Paul Mosley, Peter Moss, Thibauld Moulaert, Gareth Mulvey, Moira Munro, Randolph Myers, Marek Naczyk, Catherine Needham, Moira Nelson, Ann Netten, Gina Netto, Becci Newton, King Lun Ngok, Chris Nyland, Julia O'Connor, Javier Olivera, Michael Orton, Sharon Parkinson, Ruth Patrick, David Pearson, Helen Penn, Ole Helby Petersen, Jenny Phillimore, Martin Powell, Andrew Power, Jennifer Pribble, Chiara Pronzato, Jiwei Qian, M Ramesh, James Rees, Johanna Rickne, Michael Rosie, Antonios Roumpakis, Karen Rowlingson, Michael Roy, Hans Christian Sandlie, Katrin Schneiders, Ellen Scott, Xiaoyuan Shang, Shih-jiunn Shi, Kwang-Yeong Shin, Geri Smyth, Stefano Solari, Ian Stafford, Furio Stamati, Peter Starke, Martin Stevens, Mark Stuart, Shirley Hsiao-Li Sun, Stefanie Tan, Simon Teasdale, Judith Teichman, Hildegard Theobald, Kay Tisdall, Cecilia Tomassini, Jennifer Tomlinson, Ian Tonks, Julia Twigg, Faraz Vahid Shahidi, Rik Van Berkel, Jeroen van der Waal, Kees van Kersbergen, Barbara van Mierlo, Olaf van Vliet, Johan Vikstroem, Athina Vlachantoni, Tim Vlandas, Geoffrey Walford, Debbie Watson, Sarah White, Sue White, Adam Whitworth, Jay Wiggan, Daniel Wincott, Tobias Wiß, Sharon Wright, Lu Xiaobo, Junko Yamashita, Andrew Kam-Tuck Yip, Sam Wai-kam Yu. 